\title{
Estimativa de impactos da extração seletiva de madeiras na Amazônia utilizando dados LIDAR
}

\author{
The estimation of selective logging impact in Amazon forest using LIDAR data
}

\author{
Charton Jahn Locks ${ }^{\mathrm{I}}$, Eraldo Aparecido Trondoli Matricardi ${ }^{\mathrm{II}}$
}

\begin{abstract}
Resumo
As atividades de manejo florestal são consideradas importantes para o desenvolvimento sustentável no Brasil. Tais atividades, entretanto, exigem monitoramento rigoroso que muitas vezes é de difícil operacionalização. O mapeamento das áreas afetadas pela exploração seletiva de madeira e a mensuração dos impactos decorrentes da exploração florestal ainda são dependentes de extensos e onerosos levantamentos de campo. Neste estudo foi utilizada a tecnologia Light Detection and Ranging (LiDAR) aerotransportada para avaliação dos impactos causados pela extração seletiva de madeira em 21 Unidades de Produção Anual na Amazônia. As áreas de estudo estão localizadas nos estados de Rondônia e do Pará, dentro de Florestas Nacionais sob regime de concessão florestal federal. Foram utilizadas duas métricas derivadas da nuvem de pontos LiDAR para o mapeamento dos impactos nas florestas: a Canopy Height Model (CHM) como métrica do dossel e a Relative Density Model (RDM) como métrica do sub-bosque. Os resultados da detecção dos impactos florestais obtidos do mapeamento com dados do LiDAR são compatíveis com o levantamento realizado em campo. Estimou-se que as atividades de extração seletiva de madeiras impactaram em média 6,8\% ( $\pm 1,3 \%$, desvio padrão) da área total de sub-bosque das Unidades de Produção Anual (UPA) avaliadas e causaram incremento de $4,9 \% \pm 0,9 \%$ em área de clareiras. A tecnologia LiDAR é efetiva para o monitoramento dos impactos da extração seletiva de madeiras em áreas sob concessão florestal federal na Amazônia.
\end{abstract}

Palavras-chave: LiDAR aerotransportado; Manejo florestal sustentável; Floresta amazônica; Impactos

\begin{abstract}
Forest management activities are crucial for the sustainable development of Brazil. Those activities require, however, a strict monitoring that are often difficult to operationalize. The mapping of impacted areas by selective logging and the measurement of forest impacts because of logging operations are mostly based on extensive and costly field surveys. In this study, the Light Detection and Ranging (LiDAR) airborne technology was used to assess the impacts caused by selective logging within 21 units of forest annual production in the Amazon. The study sites are in the states of Rondônia and Pará, within National Forests under federal forestry concession. We used two metrics derived from the point cloud LiDAR for mapping forest impacts: The Canopy Height Model (CHM) and the Relative Density Model (RDM) as forest understory metric. The results of detection of forest impacts derived from the LiDAR dataset showed similar performance of field-based surveys. We estimated that selective logging activities had impacted an average of $6.8 \%( \pm 1.3 \%$, standard deviation) of the forest understory of the Annual Production Units (APU) studied and caused an increase of $4.9 \%( \pm 0.9 \%)$ in areas of forest canopy opening. The LiDAR technology showed to be effective for assessing and monitoring forest impacts of selective logging in the federal forest concessions in the Amazon.
\end{abstract}

Keywords: Airborne LiDAR; Sustainable forest management; Amazon forest; Impact

Engenheiro Ambiental, MSc., Serviço Florestal Brasileiro, SCEN, Trecho 2, Bloco G, CEP 70818-900, Brasília (DF), Brasil. charton.locks@florestal. gov.br (ORCID: 0000-0002-3048-6510)

II Engenheiro Florestal, Dr., Professor do Departamento de Engenharia Florestal, Universidade de Brasília, Campus Universitário Darcy Ribeiro, CEP 70910-900, Brasília (DF), Brasil. ematricardi@unb.br (ORCID: 0000-0002-5323-6100) 


\section{Introdução}

O mapeamento da exploração seletiva de madeiras na Amazônia, utilizando imagens de satélites ópticas tem sido realizado há vários anos. Muitos estudos demonstraram a capacidade de sensores ópticos de média resolução espacial em estimar a área explorada e a intensidade da exploração em escala de paisagem (ASNER et al., 2005; MATRICARDI et al., 2013; SOUZA et al., 2013; PINAGÉ; MATRICARDI, 2015). No entanto, os mapeamentos baseados em produtos ópticos de média resolução espacial não apresentam estimativas precisas das áreas alteradas e seus impactos (PINAGÉ; MATRICARDI, 2015).

Considerando o alto potencial da Amazônia para a economia florestal (BOMFIM et al., 2016), é necessário o aprimoramento de técnicas e sistemas para monitorar adequadamente sua exploração florestal com objetivo de reduzir a ilegalidade no setor, assegurando benefícios econômicos, sociais e ambientais do manejo florestal. Além disso, um manejo florestal mal executado pode comprometer a capacidade de recuperação do estoque comercial de madeira da floresta, além de causar uma série de impactos ambientais como, distúrbios no solo, redução da qualidade da água, da biodiversidade e dos estoques de carbono (NEPSTAD et al., 1999; MERRY et al., 2009; PUTZ et al., 2012).

As técnicas de Exploração de Impacto Reduzido (EIR) podem reduzir de 25 a 33\% dos impactos na floresta comparado com a exploração de madeiras de forma convencional (JOHNS; BARRETO; UHL, 1996). Os impactos decorrentes de uma exploração florestal são de difícil mensuração, pois podem ocorrer tanto no dossel, devido à abertura de clareiras, quanto no subbosque, em função da abertura de estradas, trilhas e pátios. A maioria dos estudos realizados em florestas tropicais, com objetivo de mensurar os impactos decorrentes do manejo florestal, mostraram-se dependentes de extensos e onerosos trabalhos de campo (ASNER; KELLER; SILVA, 2004; LENTINI; ZEWEED; HOLMES, 2009).

O uso de tecnologia de sensoriamento remoto tem a capacidade de alavancar a mensuração dos impactos de exploração florestal na Amazônia. Porém, imagens de satélite ópticas são limitadas na sua capacidade de identificar alteração no sub-bosque florestal (COOPS et al., 2007). O sistema LiDAR aerotransportado (Airborne LiDAR System - ALS) é uma tecnologia que tem a capacidade de obter informações de todo o perfil vertical da vegetação, incluindo informações do sub-bosque (JENSEN, 2009). O LiDAR vem sendo testado em inúmeras aplicações florestais, como por exemplo, estimativa de crescimento, quantificação de biomassa e estoques de carbono (NÆSSET; GOBAKKEN, 2005; ASNER et al., 2011; GIANNICO et al., 2016) e em estudos sobre degradação em florestas tropicais (D'OLIVEIRA et al., 2012; ANDERSEN et al., 2014; ELLI et al., 2016).

O princípio de funcionamento do LiDAR é baseado na utilização de um pulso de laser, disparado na direção da superfície com uma elevada frequência de repetição. Baseado no tempo de retorno, na posição do sensor e na orientação do raio é calculada a posição tridimensional do objeto (BALTSAVIAS, 1999). Sensores LiDAR de alta frequência conseguem gerar uma densa nuvem de pontos, em três dimensões, descrevendo com elevado grau de detalhes a estrutura do objeto imageado (GIONGO et al., 2010). Os produtos do LiDAR, entretanto, ainda precisam ser investigados e avaliados em aplicações voltadas ao monitoramento de impactos em florestas tropicais.

A presente pesquisa tem como objetivos mapear e mensurar o impacto à floresta remanescente após evento de manejo florestal sustentável utilizando dados LiDAR e validar, com dados de campo, o mapeamento do impacto obtido com LiDAR. 


\section{Material e método}

\section{Área de estudo}

Á área deste estudo incluiu as Florestas Nacionais (Flona) do Jamari e Jacundá, localizadas no estado de Rondônia, a Flona de Saracá-Taquera, localizada no estado do Pará, todas em regime de concessão florestal do Serviço Florestal Brasileiro. Dentro destas Flonas foram conduzidas amostras de dados LiDAR de 21 unidades de produção anual (UPA) exploradas seletivamente, intensidade média de exploração de 2,2 árvores/hectares $( \pm 0,9)$ ou $14,8 \mathrm{~m}^{3} /$ hectare $( \pm 3,6)$. A localização de cada uma das áreas de interesse deste estudo é apresentada na Figura 1, sendo nove localizadas na Floresta Nacional do Jamari-RO, oito na Floresta Nacional de Saracá-Taquera-PA e quatro na Floresta Nacional do Jacundá-RO. Na figura 1 também é possível identificar o ano de exploração de cada uma das áreas de interesse.

A Flona do Jamari, segundo a classificação fisionômica-ecológica, é predominantemente coberta por Floresta Ombrófila Densa, com algumas porções de Floresta Ombrófila Aberta, com predominância de palmeiras ou com cipós (IBGE, 2012). A Flona de Saracá-Taquera é predominantemente coberta por Floresta Ombrófila Densa, Formações Pioneiras com influência fluvial e Campinarana (IBGE, 2012). A Flona do Jacundá é coberta predominantemente por Floresta Ombrófila Aberta, com porções de Floresta Ombrófila Densa e Contatos Savana/ Floresta, Savana Parque e Savana Florestada (IBGE, 2012).

Figura 1 - Localização da área de estudo, as áreas de interesse estão distribuídas nas Florestas Nacionais (Flona) do Jamari e Jacundá, no estado de Rondônia e na Flona de Saracá-Taquera no estado do Pará.

Figure 1 - Study area locations, the areas of interest are distributed in the National Forests (Flona) of Jamari and Jacundá, in the state of Rondônia and in the Flona of Saracá-Taquera, in the state of Pará.

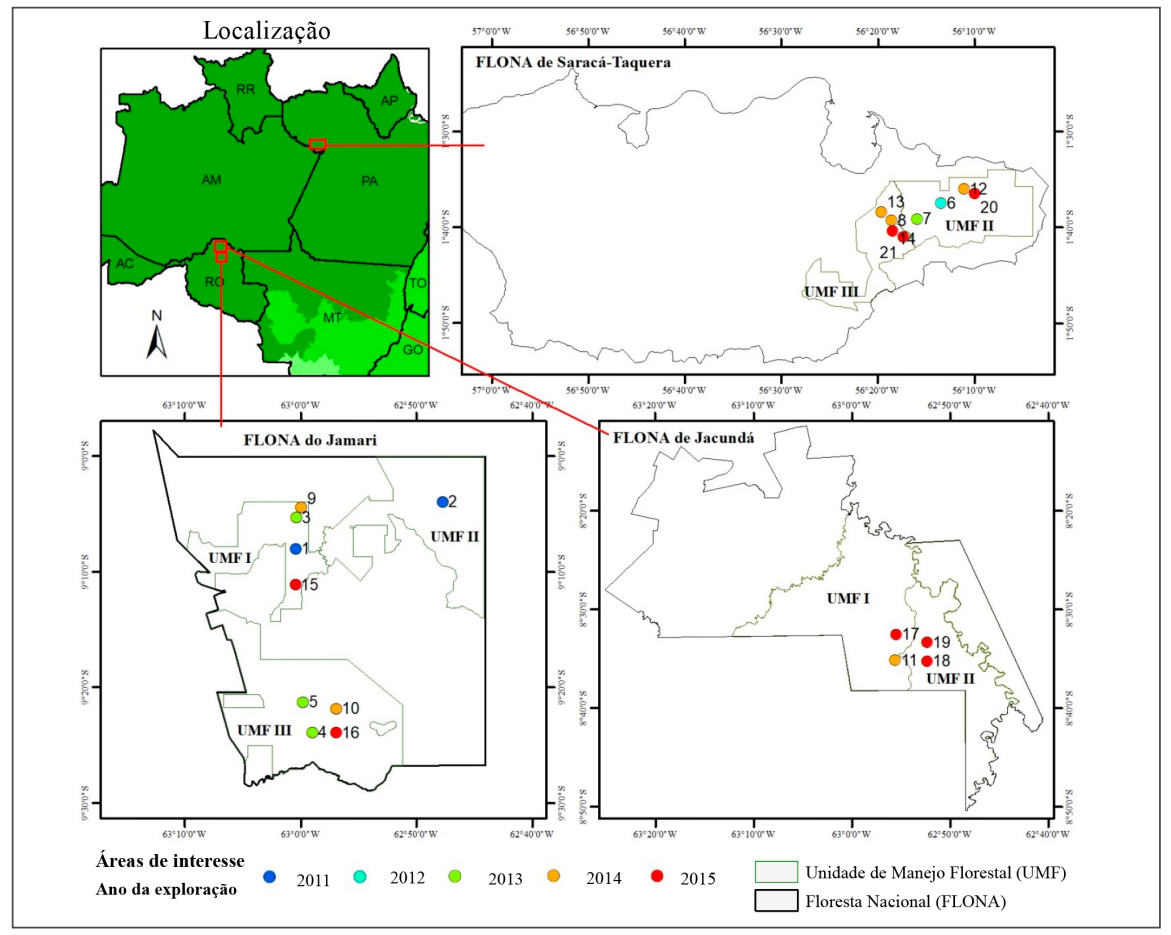

Fonte: Locks e Matricardi (2019) 


\section{Dados utilizados}

Considerando-se que as áreas de interesse foram exploradas em anos distintos, como pode ser observado na Figura 1, foi necessária a utilização de dados LiDAR coletados em diferentes anos (Tabela 1), sempre após a exploração de cada uma delas ter ocorrido, para avaliação do impacto decorrente da atividade florestal. A penas cinco áreas $(09,14,15,16$ e 20) foram imageadas com sensor LiDAR antes e após a sua exploração, permitindo assim a realização da análise de abertura de clareiras. As áreas de interesse possuem média de cobertura LiDAR de 219,0 hectares $( \pm 155,9)$. Os dados LiDAR foram disponibilizados na forma de nuvem de pontos, com extensão *las, e acompanhados do Modelo Digital do Terreno (MDT) dela derivado, com um metro de resolução espacial. A projeção e sistema de referência adotados para Flona Jamari e de Jacundá foram a UTM, fuso $20 \mathrm{~S}$ e Datum SIRGAS 2000. Para a Flona de Saracá-Taquera, adotou-se Projeção UTM, fuso $21 \mathrm{~S}$ e Datum SIRGAS 2000.

A partir do levantamento de campo realizado pela empresa concessionária, foram coletadas, para algumas áreas de interesse, informações da infraestrutura de exploração florestal utilizando equipamento Global Navigation Satellite System (GNSS) de navegação. Estes dados de campo foram exportados do equipamento GNSS no formato vetorial shapefile identificados como trilhas de arraste, estradas e pátios. Medidas de largura de cada uma destas estruturas foram coletadas em campo com trena, de forma aleatória.

Tabela 1 - Especificação técnica das coletas de dados LiDAR.

Table 1 - Technical specification of LiDAR data acquisition.

\begin{tabular}{|c|c|c|c|c|}
\hline Especificações & Coleta 2011 & Coleta 2013 & Coleta 2014 & Coleta 2015 \\
\hline Sensor LiDAR & $\begin{array}{l}\text { LaserScan, } \\
\text { Optech } 3100\end{array}$ & Optech, Orion & $\begin{array}{c}\text { Trimble, Harrier } \\
68 \mathrm{i}\end{array}$ & $\begin{array}{c}\text { LaserScan, } \\
\text { Optech } 3100\end{array}$ \\
\hline Mês da coleta & Nov. & Set./Out. & Out. & Set./Out. \\
\hline Altitude voo & $850 \mathrm{~m}$ & $850 \mathrm{~m}$ & $500 \mathrm{~m}$ & $700 \mathrm{~m}$ \\
\hline Ângulo de escaneamento & $11^{\circ}$ & $11^{\circ}$ & $15^{\circ}$ & $15^{\circ}$ \\
\hline Frequência de escaneamento & $59.8 \mathrm{kHz}$ & $67.5 \mathrm{kHz}$ & $360 \mathrm{kHz}$ & $55 \mathrm{kHz}$ \\
\hline Sobreposição lateral & $65 \%$ & $65 \%$ & $65 \%$ & $70 \%$ \\
\hline Densidade média de retornos & $25,8 \mathrm{ppm}^{2}$ & $32,9 \mathrm{ppm}^{2}$ & $49,6 \mathrm{ppm}^{2}$ & $59,2 \mathrm{ppm}^{2}$ \\
\hline Custo $(\mathbf{R} \$)$ & $49,67 /$ ha & $42,00 /$ ha & $58,05 /$ ha & $47,50 /$ ha \\
\hline
\end{tabular}

Fonte: Autores (2019)

Os dados referentes ao Inventário 100\%, realizado para cada uma das unidades de produção anual (UPA) exploradas, foram produzidos pelas empresas concessionárias obedecendo as diretrizes técnicas para apresentação de plano de manejo florestal sustentável e plano operacional anual, contidas na Norma de Execução N.ำ 1/2007 do IBAMA (IBAMA, 2007). Esses dados foram disponibilizados em formato de planilha eletrônica e arquivo vetorial.

O dado volumétrico da madeira explorada para cada uma das UPAs foi gerado pelos concessionários através da medição (cubagem) das toras, de acordo com o Guia para Medição de Produtos e Subprodutos Florestais Madeireiros das Concessões Florestais (BRASIL, 2012). Estes dados são registrados no sistema de cadeia de custódia pelas empresas concessionárias, e foram exportados do sistema para planilhas eletrônicas. 


\section{Processamento dos dados LiDAR}

O aplicativo LTKProcessor do FUSION foi utilizado para processar o conjunto de múltiplos tiles, subdivisões da nuvem de pontos LiDAR em blocos de $1.000 \times 1.000$ metros, para otimizar o processamento computacional de cada área de estudo. O resultado de cada processamento do LTK foi a geração de um conjunto de superfícies matriciais (métricas) com resolução espacial de 1 metro, contendo métricas de densidade, elevação e intensidade (MCGAUGHEY, 2010). A altitude do terreno, derivada do MDT, foi subtraída de cada retorno para remover o efeito da topografia (relevo) sobre a altura da floresta. A análise, digitalização e classificação destas métricas foi realizada no software ArcGIS.

\section{Mapeamento do impacto no sub-bosque}

A métrica LiDAR intitulada relative density model (RDM), foi utilizada para o mapeamento do impacto no sub-bosque florestal, esta métrica consiste na representação da densidade de retornos LiDAR em uma dada faixa do estrato vertical da floresta. No caso deste estudo, foi adotado o extrato de 1 a 5 metros de altura (Figura 2), pois é capaz de representar os "túneis" criados pelo trânsito de maquinário durante o arraste de toras no interior da floresta. Altos valores de RDM sugerem uma área com sub-bosque não alterado, valores próximos de zero indicam impacto no sub-bosque. Células sem informação (no data) indicam um dossel denso, capaz de impedir a penetração de feixes laser e a geração de retornos próximos ao terreno.

A partir das imagens com a métrica RDM, geradas com resolução espacial de 1 metro, foram digitalizadas manualmente na tela de um computador todas as trilhas de arraste e estradas, em formato vetorial linha, e no formato vetorial polígono todos os pátios de estocagem de cada uma das 21 áreas de interesse (4.600 hectares) deste estudo. O processo de digitalização foi feito utilizando o programa ArcGIS, em escala variável, respeitando o limite da resolução espacial das imagens. As informações de campo obtidas com GNSS não foram consultadas durante a digitalização.

Figura 2 - Cálculo do valor de densidade relativa para cada célula.

Figure 2 - Estimate of the relative density value for each cell.

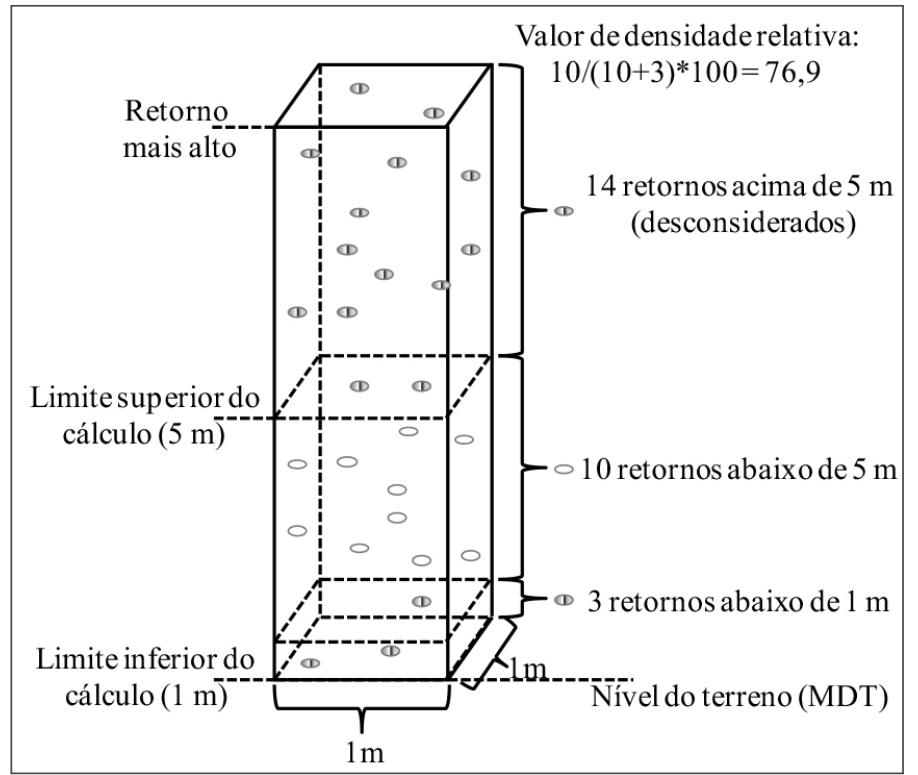

Fonte: D’Oliveira et al. (2012) 


\section{Validação do mapeamento dos impactos no sub-bosque}

Para validar o mapeamento manual do impacto no sub-bosque florestal utilizando dados do LiDAR, foi realizada a comparação com dados de campo obtidos usando GNSS de navegação modelo Garmin 76CSX. Foi comparada a quantidade, em metros lineares, de trilhas de arrastes e estradas, além da quantidade de pátios. Para avaliar a acurácia do mapeamento sobre o LiDAR, foi calculada a quantidade percentual de trilhas de arraste, estradas e pátios interceptados por zonas obtidas a partir da projeção horizontal de 5, 10 e 20 metros das feições obtidas em campo, com auxílio de GNSS (GOODCHILD; HUNTER, 1997).

\section{Mapeamento de clareiras}

Para mensurar a quantidade de clareiras em uma área objeto de manejo florestal sustentável, foi utilizada a métrica do modelo de altura do dossel (canopy height model-CHM). Esta métrica foi gerada a partir do retorno de maior altura da nuvem de pontos para cada célula de resolução espacial de 1 metro, Figura 3. A partir do CHM foram identificadas as áreas de clareiras, que de acordo com a Hunter et al. (2015), são áreas contíguas com altura inferior a 10 metros e área superior a 10 metros quadrados. O modelo de dossel foi reclassificado e vetorizado para separar os polígonos que atendessem aos dois critérios da definição de clareiras. Em seguida, foi estimada a área de clareiras por hectare para cada uma das áreas de interesse.

Como cinco das 21 áreas de interesse possuíam cobertura LiDAR prévia, a exploração florestal, foi possível detectar e comparar a quantidade de clareiras antes (naturais) e após a exploração florestal. Subtraindo a quantidade de clareiras naturais da quantidade de clareiras identificadas após a exploração foi encontrada a quantidade de clareiras decorrentes da exploração florestal.

Figura 3 - Cálculo do Modelo de Atura do Dossel.

Figure 3 - Estimate of the Canopy Height Model.

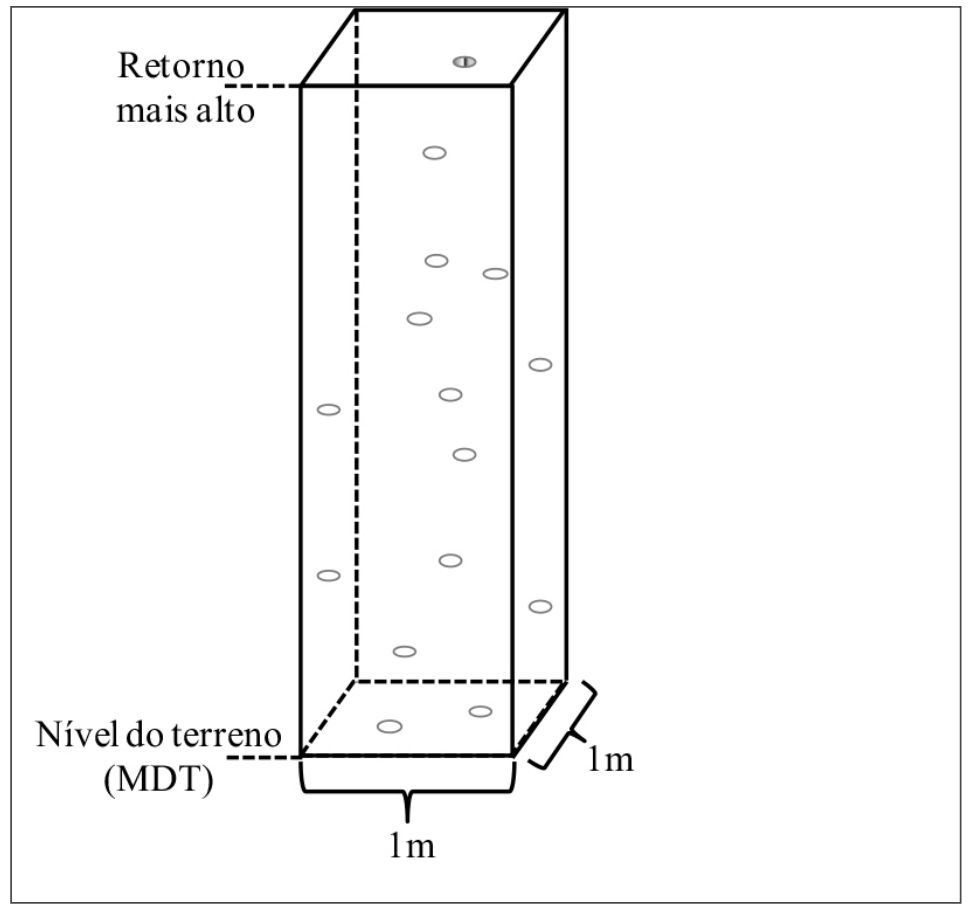

Fonte: Locks e Matricardi (2019) 


\section{Mapeamento do impacto no dossel}

Para mapear os impactos decorrentes da derrubada das árvores, realizou-se a subtração entre as métricas do modelo de altura do dossel derivados das nuvens de pontos LiDAR obtidas antes e após a exploração. Esta subtração deu origem a uma imagem diferença que foi então processada no software ERDAS IMAGINE ${ }^{\circledR}$. A rotina de processamento começou com a classificação da imagem, uma classe representando a área que não sofreu alteração de altura significativa, redução inferior a 2 metros, e outra representando a área que sofreu redução superior a 2 metros de altura. Para eliminar os ruídos presentes na imagem classificada foi utilizada a ferramenta Clump, com janela de $4 \times 4$ células. Na sequência, os agrupamentos com tamanho inferior a 129 células foram então removidos utilizando a ferramenta Sieve Table. Finalmente foi aplicado um filtro passa-baixa para suavizar o contorno dos polígonos que representam a área de dossel impactada. O fluxograma da técnica de processamento utilizada pode ser visualizado na Figura 4.

Figura 4 - Fluxograma para o mapeamento automático dos impactos da exploração seletiva no dossel florestal.

Figure 4 - Flowchart for the automatic mapping of the forest canopy impacts by selective logging.

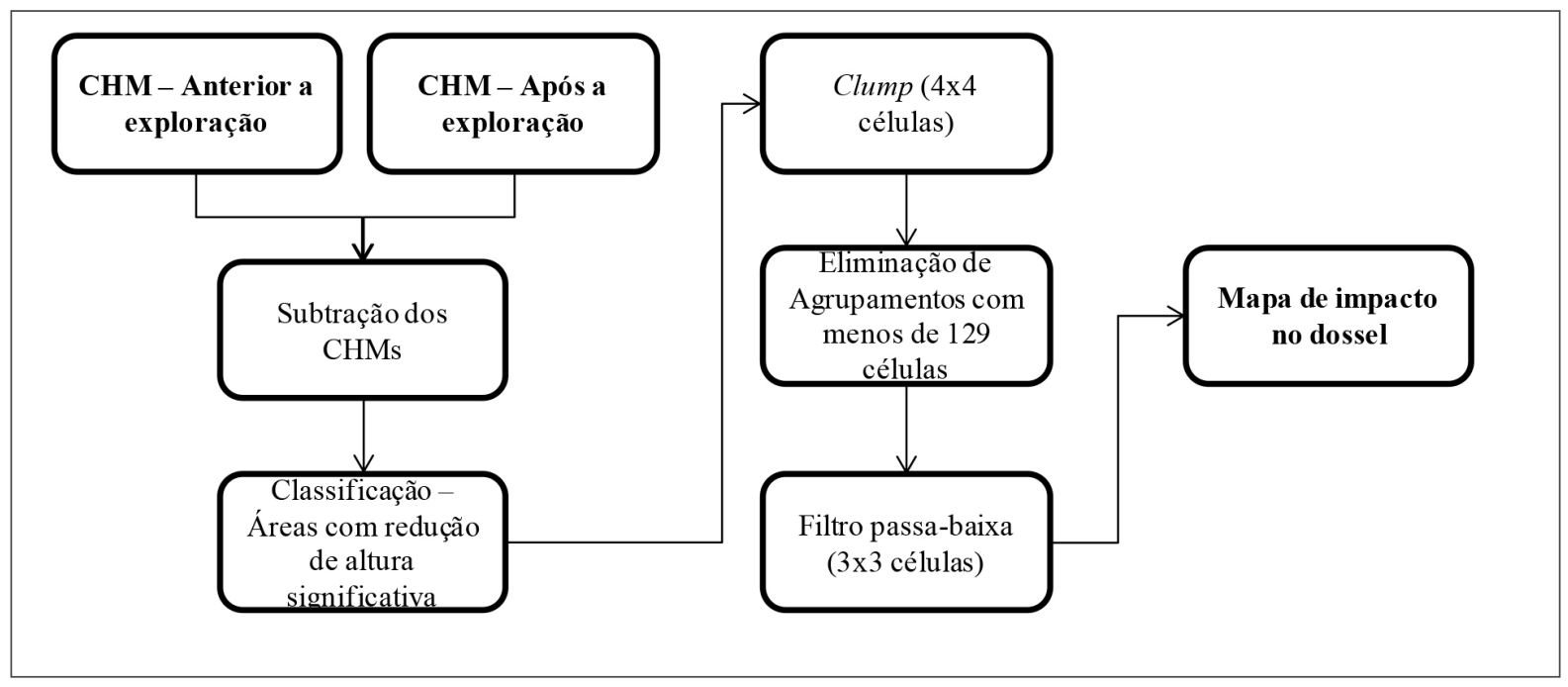

Fonte: Locks e Matricardi (2019)

\section{Validação do mapeamento de impacto no dossel}

Para validar o mapeamento dos impactos causados no dossel florestal utilizando LiDAR, os dados da classificação automática foram comparados com os dados de campo georreferenciados de todas as árvores abatidas nas áreas de interesse. Esta informação foi obtida do inventário florestal comercial (IF100\%), elaborado pelas empresas concessionárias para compor seu plano de operação anual (POA). Foi analisada a quantidade de árvores abatidas interceptadas em diferentes zonas de projeção horizontal de 5, 10 e 20 metros das clareiras identificadas nos dados LiDAR. No presente estudo, foram considerados os erros de comissão e de omissão entre os dois mapeamentos como um indicador de semelhança entre eles, não assumindo acurácia dos dados de GNSS ou LiDAR. 


\section{Resultados e discussão}

\section{Mapeamento do impacto no sub-bosque}

O padrão de exploração seletiva de madeiras na área de estudo, com estradas interligando pátio de estocagem, por sua vez irradiando trilhas de arraste pode ser observado na Figura 5(a). Os tons da imagem RDM representam em cores mais escuras, valores de baixa densidade relativa, os locais em que há escassez de alvos (neste caso vegetação), capazes de interagir com os pulsos lasers e gerarem retornos. As tonalidades mais escuras destacam tanto áreas com subbosque naturalmente mais aberto quanto as áreas impactadas pela exploração florestal. As áreas com pixels de maiores valores (cores mais claras) e de maior densidade relativa são os locais em que ocorreu maior interação entre os pulsos lasers e a vegetação, gerando maior quantidade de retornos. No presente estudo, foram consideradas como áreas não alteradas pela exploração seletiva de madeiras.

Figura 5 - Mapeamento dos impactos no sub-bosque florestal (a) a métrica RDM; (b) infraestruturas de exploração digitalizadas; (c) área total impactada.

Figure 5 - Mapping the understory impact (a) the RDM metric; (b) exploration infrastructures digitized, (c) total impacted areas.

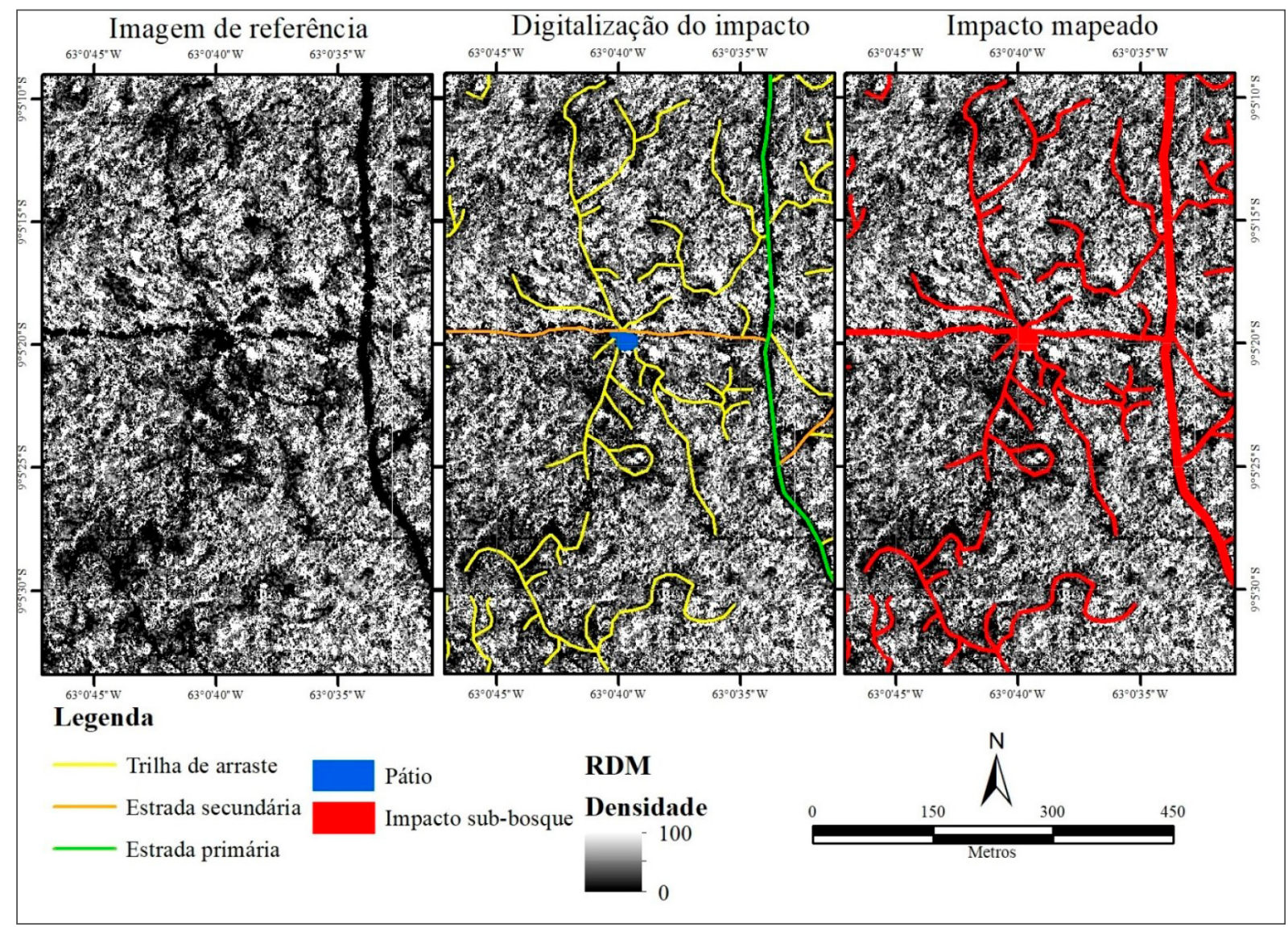

Fonte: Locks e Matricardi (2019) 
$\mathrm{Na}$ Tabela 2 é apresentada a quantidade média de infraestrutura de exploração presente no sub-bosque das áreas de interesse, identificada através de interpretação visual da métrica RDM. A quantidade média de trilhas de arraste detectada, utilizando dados LiDAR, foi de 133,8 metros por hectare $( \pm 32,5)$, Elli et al. (2016) identificaram valor ligeiramente superior, 175 metros por hectare $( \pm 32)$, porém, a intensidade média de exploração em suas áreas de estudo, em que também foram utilizadas técnicas de impacto reduzido, foi de $37 \mathrm{~m}^{3} / \mathrm{ha}( \pm 9)$, mais que o dobro das áreas avaliadas no presente estudo $14,8 \mathrm{~m}^{3} / \mathrm{ha}( \pm 3,6)$.

Tabela 2 - Infraestrutura de exploração florestal, presente no sub-bosque florestal, obtidos a partir da interpretação visual de dados LiDAR. Tri arra. = Trilha de arraste; Estr. sec. = Estrada secundária; Estr. pri. = Estrada primária.

Table 2 - Forest exploration infrastructure, in the understory, obtained by visual interpretation of LiDAR data. Tri arra. = Skid trail; Estr. Sec. = Secondary road; Estr. Pri. $=$ Primary road.

\begin{tabular}{|c|c|c|c|c|c|c|c|c|}
\hline & $\begin{array}{l}\text { Tri. arra. } \\
\quad(\mathbf{m} / \mathbf{h a})\end{array}$ & $\begin{array}{c}\text { Tri. arra. } \\
\qquad\left(\mathbf{m} / \mathbf{m}^{3}\right)\end{array}$ & $\begin{array}{c}\text { Estr. sec. } \\
(\mathbf{m} / \mathbf{h a})\end{array}$ & $\begin{array}{c}\text { Estr. sec. } \\
\left(\mathbf{m} / \mathbf{m}^{3}\right)\end{array}$ & $\begin{array}{c}\text { Estr. pri. } \\
(\mathbf{m} / \mathbf{h a})\end{array}$ & $\begin{array}{c}\text { Estr. pri. } \\
\left(\mathbf{m} / \mathbf{m}^{3}\right)\end{array}$ & $\begin{array}{c}\text { Pátio } \\
\left(\mathbf{m}^{2} / \mathbf{h a}\right)\end{array}$ & $\begin{array}{c}\text { Pátio } \\
\left(\mathbf{m}^{2} / \mathbf{m}^{3}\right)\end{array}$ \\
\hline $\begin{array}{l}\text { Média } \pm \\
\text { Desvio } \\
\text { Padrão }\end{array}$ & $\begin{array}{r}133,8 \\
\pm 32,5\end{array}$ & $\begin{array}{r}9,1 \\
\pm 1,6\end{array}$ & $\begin{array}{r}18,7 \\
\pm 4,4\end{array}$ & $\begin{array}{r}1,3 \\
\pm 0,4\end{array}$ & $\begin{array}{r}7,7 \\
\pm 5,0\end{array}$ & $\begin{array}{r}0,6 \\
\pm 0,4\end{array}$ & $\begin{array}{r}49,7 \\
\pm 18,4\end{array}$ & $\begin{array}{r}3,4 \\
\pm 1,1\end{array}$ \\
\hline
\end{tabular}

Fonte: Autores (2019)

A média de impacto no sub-bosque em florestas sob concessão florestal federal foi de $6,8 \%$ $( \pm 1,3 \%)$. Esta média é próxima à observada em estudos baseados em levantamentos de campo em florestas tropicais submetidas à exploração de impacto reduzido, cujos resultados variaram de 4,6\% a 8,6\% (JOHNS; BARRETO; UHL, 1996; PEREIRA et al., 2002; ASNER; KELLER; SILVA, 2004; LENTINI; ZEWEED; HOLMS, 2009). O maior percentual de área alterada no sub-bosque florestal para implantação de infraestrutura de exploração é decorrente da abertura de trilhas de arraste, aproximadamente $69,9 \%( \pm 7,9 \%)$, seguido pela construção de estradas secundárias $14,4 \%$ $( \pm 3,8 \%)$, estradas primárias $8,3 \%( \pm 6,9 \%)$ e pátios $7,3 \%( \pm 2,3 \%)$. As trilhas de arraste constituem o tipo de infraestrutura florestal que, em quantidade de área, mais impacta o sub-bosque florestal, entretanto, são consideradas infraestrutura temporária e sua identificação, utilizando dados de sensores remotos, fica comprometida dois a três anos após a exploração da área (MATRICARDI et al., 2013).

Apenas três das 21 áreas avaliadas apresentaram impacto superior ao definido pelo SFB (8\% da área total da UPA), estas áreas apresentaram intensidade média de exploração de 19,4 m³/ ha $( \pm 0,6)$, superior à média geral deste estudo, mas ainda consideravelmente inferior ao máximo permitido para um ciclo de corte de 30 anos, por volta de $25 \mathrm{~m}^{3} /$ ha. Assumindo que os impactos à floresta estão positivamente relacionados ao número de árvores/hectare (Figura 6a) e ao volume explorado $\left(\mathrm{m}^{3} / \mathrm{ha}\right)$ (Figura 6b) e que existe espaço para aumento da intensidade de exploração nas áreas sob concessão, deve-se considerar, por parte dos concessionários, o uso de modelos geoespaciais de planejamento associados a informações técnicas e métodos computacionais, que, de acordo com Emmert (2014), podem otimizar a construção da infraestrutura de exploração, reduzindo assim os impactos causados no sub-bosque e os custos da produção florestal. 
Figura 6 - Correlação entre impacto no sub-bosque e IEX = Intensidade de Exploração, relação entre impacto no sub-bosque e a quantidade de árvores exploradas por hectare (a); e relação entre impacto no sub-bosque e volume explorado, em metros cúbicos por hectare (b).

Figure 6 - Correlation between impact in the understory and IEX = Intensity of Exploration, relationship between impact in the understory and the amount of trees harvested per hectare

(a); and relationship between understory impact and volume exploited, in cubic meters per hectare (b).

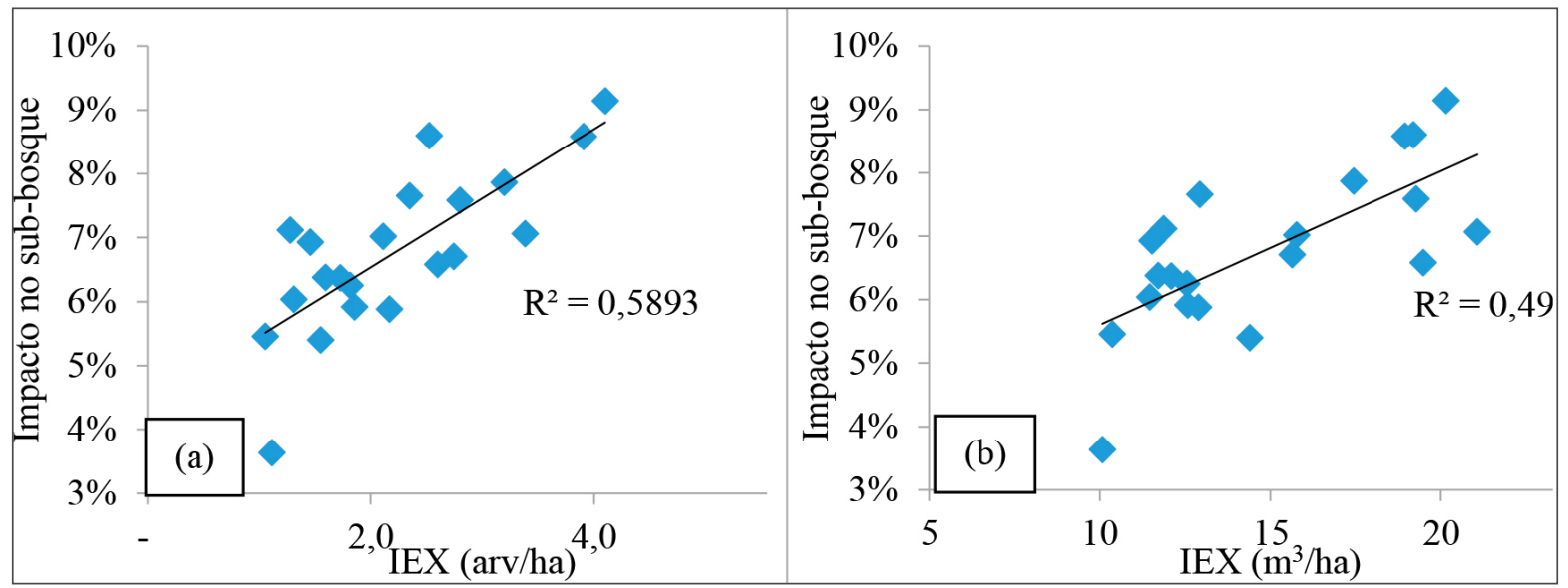

Fonte: Locks e Matricardi (2019)

\section{Validação do mapeamento dos impactos no sub-bosque}

A comparação entre o mapeamento manual de impacto no sub-bosque utilizando dados LiDAR e o mapeamento de campo com GNSS de navegação demonstra que as ambas as técnicas apresentam resultados semelhantes (Tabela 3). A diferença quantitativa do mapeamento de cada uma das infraestruturas de exploração, com exceção das trilhas de arraste, foi muito próxima de zero. O mapeamento LiDAR acabou superestimando em $9,4 \%$ o total de trilhas de arraste, para a área-controle, quando comparado com dados de GNSS. Uma das razões pode ser a inclusão de parte das áreas de clareira/galhada, que também perturbam o sub-bosque, como continuação das trilhas de arraste. Outra razão é a dificuldade de mapeamento em campo de todas as trilhas de arraste depois de encerrada a operação, como feito neste estudo. Elli et al. (2016) também encontraram um valor $14 \%$ superior no mapeamento de trilhas de arraste e áreas de queda de árvore utilizando LiDAR quando comparado com dados de campo obtidos com GNSS de navegação.

Como relação á acurácia posicional de até 20 metros para o GNSS, o mapeamento LiDAR se equipara ao mapeamento de campo. Locais de dossel mais aberto, como estradas primárias e pátios, foram os locais que apresentaram maior acurácia posicional, corroborando com a afirmação de que um dossel fechado contribui para o aumento do erro posicional do GNSS. 


\section{Tabela 3 - Comparação do mapeamento de impacto no sub-bosque realizado em campo} (GNSS) e utilizando dados LiDAR.

Table 3 - Comparison of understory impact mapping in the field (GNSS) and using LiDAR data.

\begin{tabular}{lcccccc}
\hline \multirow{1}{*}{ Infraestrutura } & Mapeamento - & Mapeamento - & Diferença & \multicolumn{3}{c}{ Sobreposição (\%) } \\
& GNSS & LiDAR & & $\mathbf{5 m}$ & $\mathbf{1 0 m}$ & $\mathbf{2 0 m}$ \\
\hline Trilha de arraste $(\mathbf{m})$ & $11.124,9$ & $12.174,5$ & $9,4 \%$ & $36,0 \%$ & $65,0 \%$ & $86,5 \%$ \\
Estr. secundária $(\mathbf{m})$ & $12.260,2$ & $12.281,4$ & $0,2 \%$ & $70,2 \%$ & $92,6 \%$ & $97,2 \%$ \\
Estr. primária $(\mathbf{m})$ & $12.057,3$ & $12.042,0$ & $-0,1 \%$ & $53,5 \%$ & $81,3 \%$ & $99,0 \%$ \\
Pátio $($ un) & 35 & 35 & $0,0 \%$ & $88,6 \%$ & $91,4 \%$ & $100,0 \%$ \\
\hline
\end{tabular}

Fonte: Locks e Matricardi (2019)

\section{Mapeamento de clareiras}

Conforme apresentado na Figura 7, a partir da métrica CHM é possível identificar a quantidade, altura e formato da copa de cada uma das árvores emergentes da floresta. $\mathrm{Na}$ figura 7(a), obtida antes da exploração, foi sobreposta a camada de clareiras (polígonos laranja), consideradas como regiões com menos de 10 metros de altura e mais de 10 metros quadrados de área, extraídas da própria métrica de CHM. O padrão de clareiras após uma exploração florestal pode ser observado na Figura 7(b).

Figura 7 - Clareiras na floresta antes (a), e após (b) a exploração de madeira via Manejo Florestal Sustentável.

Figure 7 - Forest gaps before (a), and after (b) the timber exploration by Sustainable Forest Management.

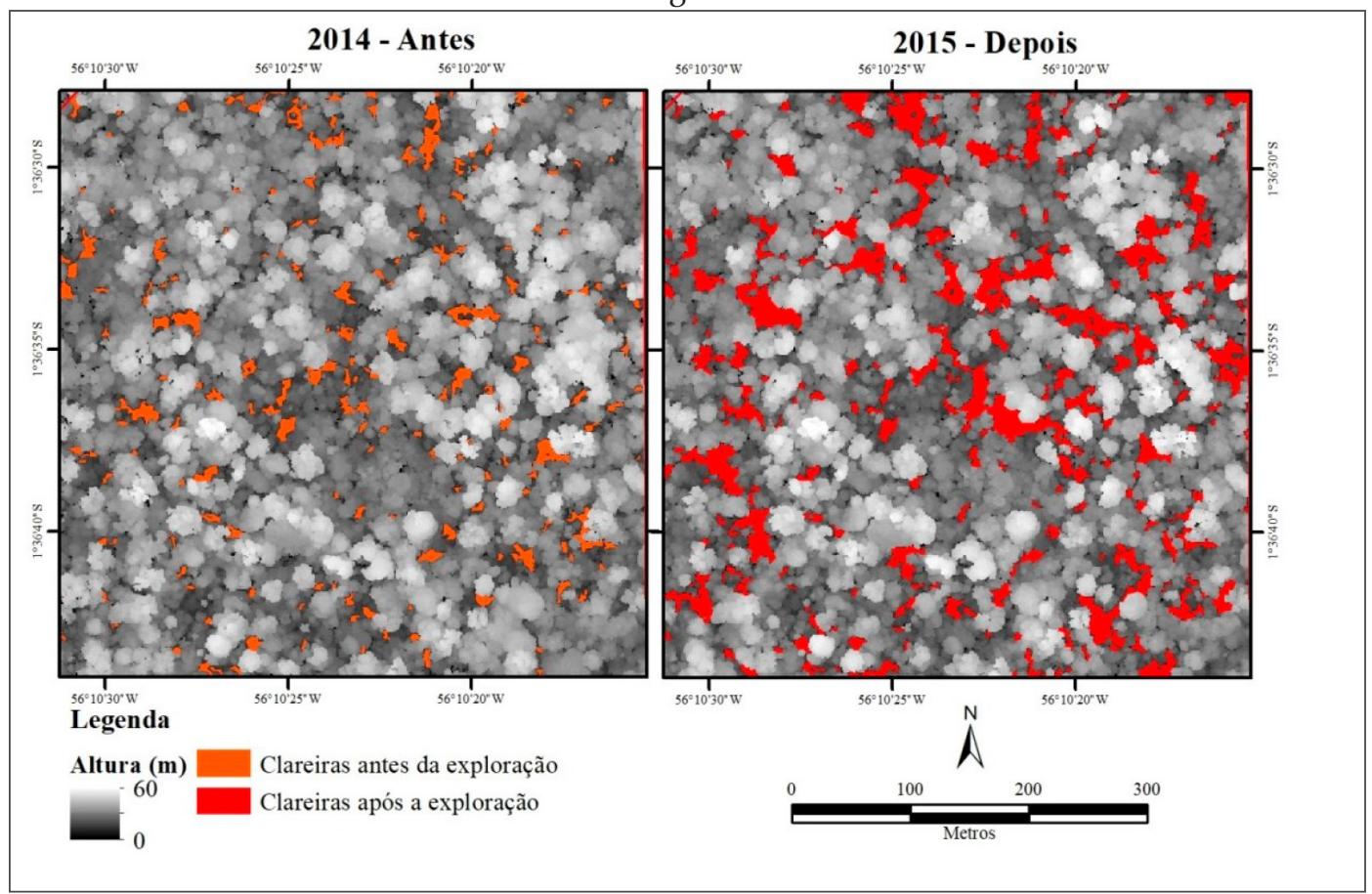

Fonte: Locks e Matricardi (2019) 
As áreas deste estudo apresentaram, após sua exploração madeireira, uma quantidade média de clareiras de 7,8\% ( $\pm 1,4 \%)$. Mas, nem toda clareira identificada pode ser considerada como resultado da exploração, uma boa parte delas ocorre naturalmente, assim como visto por Hunter et al. (2015), que ao avaliarem as clareiras naturais presentes em área de floresta primária na Floresta Nacional do Tapajós-PA e na Reserva Ducke-AM, utilizando dados LiDAR, encontraram valores de $4,8 \%$ e $2 \%$ respectivamente.

Para as cinco áreas que tiveram o mapeamento LiDAR realizado antes e após a sua exploração foi realizada a mensuração da quantidade de clareiras nestes dois momentos. A área ocupada por clareiras naturais nas parcelas avaliadas foi, em média, de $3,7 \%( \pm 1,3 \%)$, estas mesmas áreas apresentaram uma quantidade de clareiras da ordem de $8,6 \%( \pm 1,7 \%)$ após o manejo florestal. Dessa maneira, a média de abertura de clareiras em função da exploração florestal seletiva de madeira para estas áreas é da ordem de 4,9\% ( \pm 0,9\%). Matricardi et al. (2013), utilizando imagens dos satélites Landsat TM e ETM+, mensuraram a alteração no dossel florestal em 5,0\% ( $\pm 0,4 \%)$ para áreas recém-manejadas na Amazônia brasileira, a técnica utilizada pelos autores, denominada modified soil adjusted vegetation index aerosol free (MSAVIaf), é dependente da quantidade de solo exposto na região florestal, ou seja, das áreas de clareiras na floresta, o que ajuda a explicar a similaridade dos resultados mesmo considerando técnicas e sensores tão diferentes ao deste estudo. O limite estabelecido pelo SFB, em seus contratos de concessão, para abertura de clareiras, é de $10 \%$ do total da área manejada, ou seja, as áreas avaliadas estão dentro dos limites de impactos estabelecidos pelo SFB.

A localização geográfica de cada uma das árvores abatidas nas florestas sob concessão federal é uma informação disponível no Serviço Florestal Brasileiro, mas mensurar a área afetada por sua queda não havia sido feito até o momento. Dentro das cinco áreas de interesse deste estudo, nas quais foi possível quantificar as clareiras decorrentes da exploração, foram derrubadas 1.454 árvores. Para estas áreas foi encontrada uma média de $186,6 \mathrm{~m}^{2}$ árvore $( \pm 33,5)$ e $27,9 \mathrm{~m}^{2} / \mathrm{m}^{3}$ explorado $( \pm 2,6)$. Asner, Keller e Silva (2004) ao mensurar em campo a abertura de clareiras em quatro áreas objeto de exploração de impacto reduzido encontraram o valor de $175 \mathrm{~m}^{2} /$ árvore $( \pm 42)$, bem próximo ao encontrado neste estudo. Explorações de madeira que não utilizaram técnicas de impacto reduzido, também avaliadas por Asner, Keller e Silva (2004), apresentaram abertura de clareiras muito superior, da ordem de $328 \mathrm{~m}^{2} /$ árvore $( \pm 143)$.

\section{Mapeamento do impacto no dossel}

A abertura de clareiras pode ser considerada um dos parâmetros de impacto à floresta objeto de manejo florestal. Porém, a derrubada das árvores não gera apenas uma clareira. Considerando que o extrato vertical de uma floresta nativa é composto de árvores de diferentes tamanhos e alturas, a derrubada de uma árvore, além de abrir uma clareira expõe a copa de outras árvores. Nesse sentido, foram utilizados os dados LiDAR multitemporais (antes e após a exploração) para estimar a total alteração no dossel florestal decorrente do manejo (Figura 8). O impacto total no dossel florestal, definido como a área com redução superior a dois metros de altura após exploração florestal, é superior à quantidade de clareiras, na média $12,8 \%( \pm 3,0 \%)$ da área explorada apresenta redução no dossel. 
Figura 8 - Alterações no dossel florestal decorrentes do manejo florestal sustentável, (a) Modelo de Altura do Dossel antes da exploração; (b) Modelo de Altura do Dossel após a exploração; (c) imagem diferença; (d) resultado da classificação, impacto no dossel florestal representado pelos polígonos vermelhos.

Figure 8 - Changes in the forest canopy due the sustainable forest management, (a) Canopy Height Model before exploration; (b) Canopy Height Model after exploration; (c) difference image; (d) classification result, impact in the forest canopy represented by the red polygons.

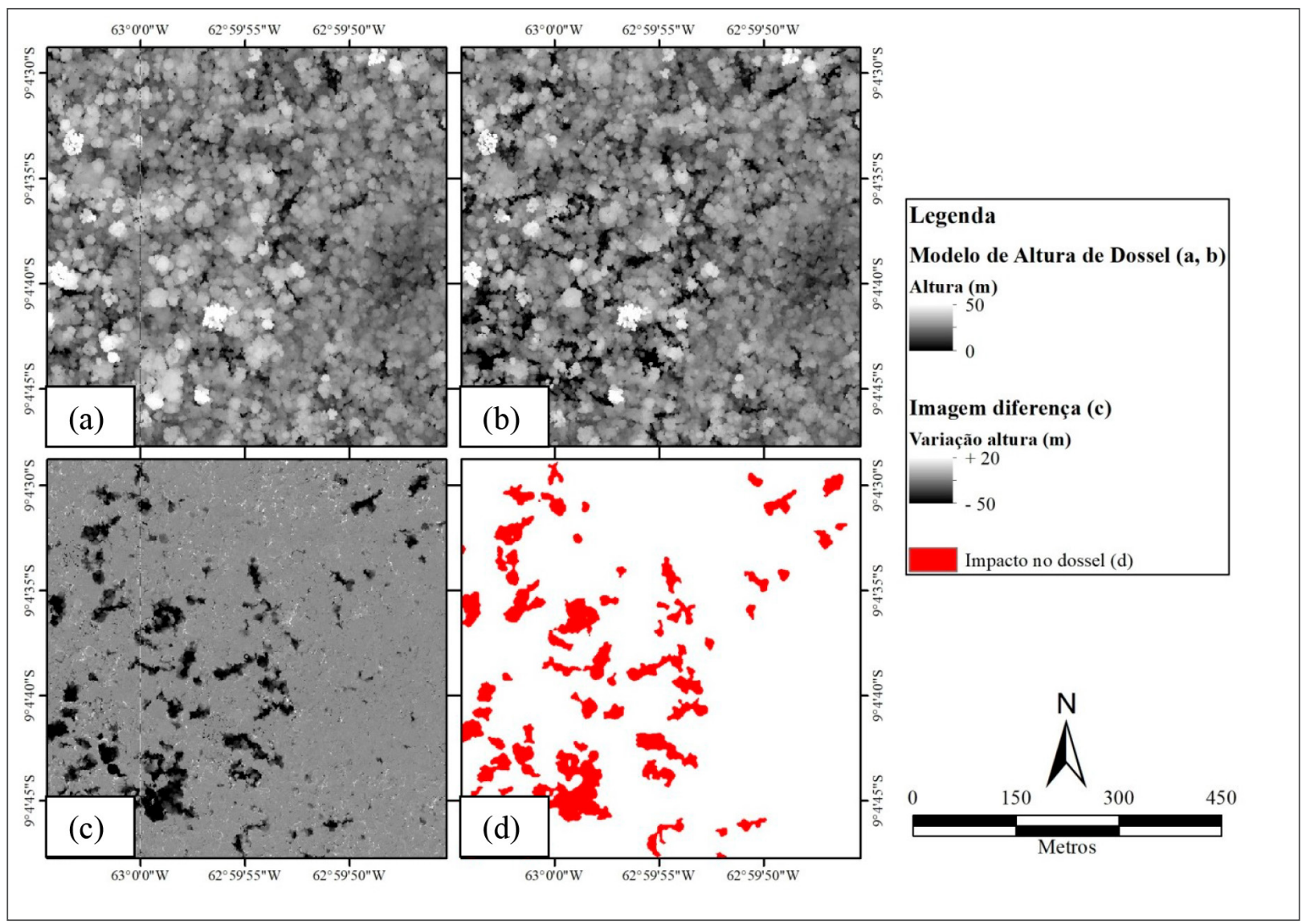

Fonte: Locks e Matricardi (2019)

\section{Validação do mapeamento do impacto no dossel}

Do total de 1.454 árvores exploradas nas cinco áreas de interesse deste estudo, praticamente todas elas $(93,3 \%)$ foram interceptadas pelos polígonos de impacto no dossel identificados no mapeamento LiDAR, considerado um erro posicional de até 20 metros do equipamento GNSS. Esse resultado indica a existência de relação espacial entre o impacto no dossel identificado a partir de dados LiDAR e a derrubada de árvores em campo.

\section{Conclusões}

É possível monitorar com boa acurácia a exploração seletiva de madeira utilizando dados LiDAR, incluindo a mensuração dos impactos no sub-bosque e dossel florestal na área explorada. Os resultados das estimativas utilizando dados LiDAR são similares as medições feitas em campo. 
Assumindo um erro posicional de até 20 metros, mais de $90 \%$ da infraestrutura de exploração florestal mapeada com LiDAR é coincidente com dados coletados em campo com GNSS. Considerando o mesmo erro posicional, $93,3 \%$ das árvores abatidas são interceptadas pelo impacto no dossel mapeado com LiDAR.

A exploração realizada nas áreas de concessão florestal federal do presente estudo, no que diz respeito ao impacto a floresta remanescente, pode ser considerada como EIR. O impacto médio identificado no sub-bosque da floresta remanescente foi de $6,8 \%( \pm 1,3 \%)$. O percentual de clareiras encontradas nas áreas exploradas foi de $7,8 \%( \pm 1,4 \%)$, sendo que para as áreas com cobertura LiDAR multitemporal, a abertura de clareiras decorrentes do manejo florestal (descontado as clareiras naturais presentes antes da exploração) foi de $4,9 \%( \pm 0,9 \%)$.

Observou-se correlação entre o impacto à floresta remanescente e a intensidade de exploração, indicando que os baixos valores de impacto ao sub-bosque são, em parte, explicados pela baixa intensidade média de exploração, $14,8 \mathrm{~m}^{3} / \mathrm{h}$. Dessa maneira, deve ser preocupação constante dos concessionários do SFB aperfeiçoar suas técnicas de manejo para que, no caso de exploração plena da área (exploração de até $25,8 \mathrm{~m}^{3} /$ ha para um ciclo de corte de 25 anos), o impacto não ultrapasse o limite contratual de $8 \%$.

Mensurar o impacto à floresta remanescente, utilizando dados LiDAR, pode contribuir com o monitoramento da atividade madeireira na Amazônia brasileira, pois permite não apenas identificar com precisão as áreas manejadas, mas também qualificar, de maneira objetiva, a execução de um Plano de Manejo Florestal.

\section{Referências}

ANDERSEN, H. E. et al. Monitoring selective logging in western Amazonia with repeat LiDAR flights. Remote Sensing of Environment, New York, v. 151, p. 157-165, 2014.

ASNER, G. P. et al. Selective logging in the Brazilian Amazon. Science, Washington, n. 310, p. 480-482, 2005.

ASNER, G. P. et al. A universal airborne LiDAR approach for tropical forest carbon mapping. Oecologia, Berlin, v. 168, n. 4, p. 1147-1160, 2011.

ASNER, G. P.; KELLER, M.; SILVA, J. N. M. Spatial and temporal dynamics of forest canopy gaps following selective logging in the eastern Amazon. Global Change Biology, [s. l.], v. 10, p. 765-783, 2004.

BALTSAVIAS, E. P. A comparison between photogrammetry and laser scanning. ISPRS Journal of Photogrammetry and Remote Sensing, [s. l.], v. 54, n. 2/3, p. 83-94, 1999.

BOMFIM, S. L. et al. Índice de clima econômico para concessões florestais. Scientia Florestalis, Piracicaba, v. 44, n. 10, p. 331-342, 2016.

BRASIL. Ministério do Meio Ambiente. Serviço Florestal Brasileiro. Guia para medição de produtos e subprodutos florestais madeireiros das concessões florestais. Brasília, 2012. 48 p.

COOPS, N. C. et al. Estimating canopy structure of Douglas-fir forest stands from discretereturn LIDAR. Trees, [s. l.], v. 21, n. 3, p. 295-310, 2007.

D’OLIVEIRA, M. V. N. et al. Estimating forest biomass and identifying low-intensity logging areas using airborne scanning LiDAR in Antimary State Forest, Acre State, Western Brazilian Amazon. Remote Sensing of Environment, New York, v. 124, p. 479-491, 2012.

ELLI, P. et al. Mapping selective logging impacts in Borneo with GPS and airborne LiDAR. Forest Ecology and Management, Amsterdam, v. 365, p. 184-196, 2016.

EMMERT, F. Combinação de dados de campo e métodos computacionais para o planejamento de exploração florestal na Amazônia. 2014. Tese (Doutorado) - Universidade de Brasília, Brasília, 2014. 
GIANNICO, V. et al. Estimating stand volume and above-ground biomass of urban forests using LiDAR. Remote Sensing, Basel, v. 8, p. 14, 2016.

GIONGO, M. et al. LiDAR: princípios e aplicações florestais. Pesquisa Florestal Brasileira, Colombo, v. 30, n. 63, p. 231-244, 2010.

GOODCHILD, M. F.; HUNTER, G. J. A simple positional accuracy measure for linear features. International Journal of Geography Information Science, London, v. 11, p. 299-306, 1997.

HUNTER, M. O. et al. Structural dynamics of tropical moist forest gaps. PLOS One, San Francisco, v. 10, p. 19, 2015.

IBGE. Manual técnico da vegetação brasileira. 2. ed. Rio de Janeiro: IBGE, 2012. 271 p.

IBAMA. Norma de Execução № 1, de 24 de abril de 2007. Diretrizes técnicas para elaboração dos planos de manejo florestal sustentável - PMFS. Diário Oficial da União: seção 1, Brasília, DF, p. 405,30 abr. 2007.

JENSEN, J. R. Sensoriamento remoto do ambiente: uma perspectiva em recursos terrestres. São José dos Campos: Parêntese, 2009. 672 p.

JOHNS, J. S.; BARRETO, P.; UHL, C. Logging damage during planned and unplanned logging operations in the eastern Amazon. Forest Ecology and Management, Amsterdam, v. 89, p. 5977, 1996.

LENTINI, M. W.; ZEWEED, J. C.; HOLMES, T. P. Measuring Ecological Impacts from Logging in Natural Forests of the Eastern Amazônia as a Tool to Assess Forest Degradation. Rome: [s. n.], 2009. (Forest Resources Assessment Working Paper, 165).

MATRICARDI, E. A. T. et al. Assessment of forest disturbances by selective logging and forest fires in the Brazilian Amazon using Landsat data. International Journal of Remote Sensing, London, v. 34, n. 4, p. 1057-1086, 2013.

MCGAUGHEY, R. J. FUSION/LDV: Software for LIDAR data analysis and visualization. [S. l.]: United States Department of Agriculture, Forest Service, Pacific Northwest Research Station, 2010.

MERRY, F. et al. Balancing conservation and economic sustainability: the future os the Amazon timber industry. Environmental Management, Springer, v. 44, p. 395-407, sep. 2009.

NÆSSET, E.; GOBAKKEN, T. Estimating forest growth using canopy metrics derived from airborne laser scanner data. Remote Sensing of Environment, New York, v. 96, p. 453-465, 2005.

NEPSTAD, D. C. et al. Large-scale impoverishment of Amazonian forests by logging and fire. Nature, London, n. 398, p. 505-508, 1999.

PEREIRA, R. et al. Forest canopy damage and recovery in reduced-impact and conventional selective logging in eastern Para, Brazil. Forest Ecology and Management, Amsterdam, v. 168, p. 77-89, 2002.

PINAGÉ, E. R.; MATRICARDI, E. A. T. Detecção da Infraestrutura para Exploração Florestal em Rondônia Utilizando Dados de Sensoriamento Remoto. Floresta e Ambiente, Rio de Janeiro, v. 22, n. 3, p. 377-390, 2015.

PUTZ, F. E. et al. Sustaining conservation values in selectively logged tropical forests: the attained and the attainable. Conservation Letters, Hoboken, v. 5, p. 296-303, 2012.

SOUZA, C. M. et al. Ten-Year Landsat Classification of Deforestation and Forest Degradation in the Brazilian Amazon. Remote Sensing, Basel, n. 5, p. 5493-5513, 2013. 